\title{
Eslicarbazepine Acetate
}

\begin{tabular}{l|l}
\hline Rosuvastatin & Rosuvastatin does not affect the pharmacokinetics of eslicarbazepine [1]
\end{tabular}

\section{Reference}

1. Gidal BE, Mintzer S, Schwab M, Schutz R, Kharidia J, Blum D, Grinnell T, Sunkaraneni $\mathrm{S}$. Evidence for a pharmacokinetic interaction between eslicarbazepine and rosuvastatin: potential effects on xenobiotic trasporters. Epileepsy Res. 2017;135:64-70. 